\title{
Les techniques de sculpture dans l'Antiquité tardive et le haut Moyen Âge
}

Sculptural techniques in Late Antiquity and the Early Middle Ages

\section{Thierry Gregor et Anne-Bénédicte Mérel-Brandenburg}

\section{OpenEdition}

\section{Journals}

Édition électronique

URL : https://journals.openedition.org/cel/19765

DOI : $10.4000 /$ cel. 19765

ISSN : 2262-208X

\section{Éditeur}

École du Louvre

\section{Référence électronique}

Thierry Gregor et Anne-Bénédicte Mérel-Brandenburg, «Les techniques de sculpture dans l'Antiquité tardive et le haut Moyen Âge », Les Cahiers de l'École du Louvre [En ligne], 17 | 2021, mis en ligne le 22 novembre 2021, consulté le 13 décembre 2021. URL : http://journals.openedition.org/cel/19765 ; DOI : https://doi.org/10.4000/cel.19765

Ce document a été généré automatiquement le 13 décembre 2021.

\section{c) (†) $\odot$}

Les Cahiers de l'École du Louvre sont mis à disposition selon les termes de la licence Creative Commons Attribution - Pas d'Utilisation Commerciale - Pas de Modification 4.0 International. 


\section{Les techniques de sculpture dans l'Antiquité tardive et le haut Moyen Âge}

Sculptural techniques in Late Antiquity and the Early Middle Ages

Thierry Gregor et Anne-Bénédicte Mérel-Brandenburg

1 Les études sur les techniques de la pierre et son approche objective tant au niveau de la taille que de la sculpture sont peu présentes dans les ouvrages. Les références incontournables sont les travaux de Jean-Claude Bessac ${ }^{1}$, d'Étienne Cunrath ${ }^{2}$ et des compagnons ${ }^{3}$. Ces sommes très spécifiques traitent des outils et de leurs usages, mais peu des techniques de sculpture. Or, l'observation attentive des œuvres sculptées à des fins architecturales, liturgiques ou funéraires, produites au cours de l'Antiquité tardive et du haut Moyen Âge, permet d'observer des détails caractéristiques informant sur les conditions d'exécution en fonction du support. Les traces d'outils discernables sur leurs parements autorisent à envisager les procédés mis en œuvre par les sculpteurs ainsi que leurs gestes. Une analyse méthodologique appliquée systématiquement pour le travail de la pierre apporte des données capitales à la compréhension de la chaîne opératoire et des savoir-faire hérités de l'Antiquité classique, et renouvelle la connaissance de la production sculptée.

\section{Matériaux et propriétés physiques}

2 Les types de pierre sont multiples et leurs propriétés physiques déterminent les méthodes et le choix des outils élaborés par le sculpteur dans le but d'exécuter une commande. Un tuffeau, pierre calcaire très tendre, ne se taille pas ou ne se sculpte pas avec les mêmes moyens qu'un granit, pierre particulièrement dure.

3 Sans pratiquer d'analyses pétrographiques poussées, les différences de densité se perçoivent en fonction de la sonorité des pierres, soit en tapotant avec un objet dur la surface du bloc, soit lors de la taille de blocs de composition différente avec un même 
outil, un ciseau gravelet par exemple : le son est "grave " si la densité est faible, à l'exemple du tuffeau, et le son est «aigu » si la pierre a une forte densité, comme les marbres ou les granits. La tonalité informe sur la difficulté de mise en œuvre, dans la mesure où la taille du calcaire est plus aisée que celle du marbre.

\section{Les outils}

4 Que le sculpteur soit expérimenté ou novice, le choix des outils est essentiel. Si les outils ne sont pas adaptés à l'activité, taille de pierre ou sculpture, la tâche se révèle complexe, voire impossible à effectuer correctement. Par conséquent l'exécutant choisit ses outils en fonction des matériaux, avec des variations suivant les époques.

Trois types d'outils sont utilisés pour la taille de pierre et la sculpture. Le premier type d'outil, les outils à percussion lancée, correspond à des outils tenus à une ou deux mains, comme les têtus, les pics (fig. 1.a), les taillants. L'artisan, lors des opérations de dégrossi, envoie la partie active de l'outil percuter le bloc, pour enlever de la matière. Les traces de cette catégorie d'outil ne sont en général pas visibles sur les blocs, car elles ont disparu la plupart du temps au fur et à mesure des étapes de taille. Or, ce type d'outil est employé massivement pour la taille de la pierre, en particulier pour la réalisation des cuves de sarcophage, pour laquelle les exécutants possédaient une dextérité parfois confondante. Le second type, les outils à percussion posée avec percuteur, est utilisé pour enlever de la matière lors des étapes d'approche et de finition. Les broches, ciseaux (fig. 1.b) et gradines, tenus dans une main et percutés par un maillet ou une massette, permettent d'approcher ou de réaliser la surface finie. Le dernier type regroupe les outils à percussion posée sans percuteur, parfois discernables sur les parements des pierres. Les râpes, ripes avec dents ou sans dents (fig. 1.c), outils de finition, laissent quelques traces perceptibles en lumière rasante. 
Fig. 1. Outils
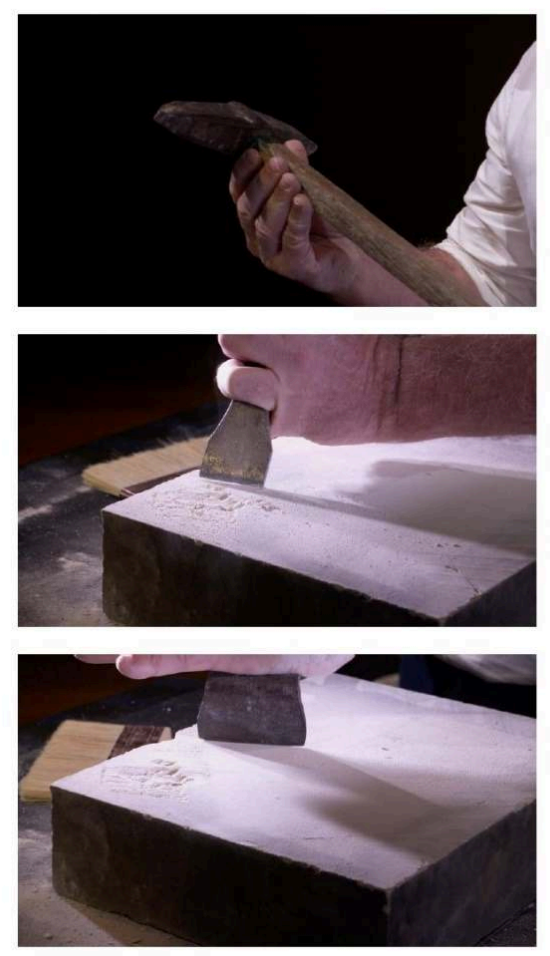

a. Pic ${ }^{4}$. b. Ciseau c. Ripe.

(c) musée du Louvre

6 Suivant la nature des pierres et les périodes, le nombre d'outils et les méthodes diffèrent. Les blocs sculptés au cours de l'Antiquité tardive et du haut Moyen Âge analysés dans les collections de Poitiers et du musée du Louvre semblent présenter moins de traces d'outils distinctes - principalement des outils à percussion posée avec percuteur et outils à percussion posée sans percuteur - que ceux sculptés dans l'Antiquité ou à partir du X $X^{e}$ siècle, qu'il s'agisse d'une surface plane ou d'un volume.

\section{Techniques de sculpture et gestes}

$7 \quad$ Le couvercle de sarcophage $\mathrm{n}^{\circ} 41$, conservé au musée lapidaire du baptistère Saint-Jean de Poitiers ${ }^{5}$, en calcaire local et daté du VII ${ }^{\mathrm{e}}$ siècle, a été mis au jour par Camille de La Croix lors des fouilles d'Antigny (Vienne) en 1884-1886 (fig. 2.a). Il s'intègre dans un groupe de sarcophages à couvercle plat ou légèrement bombé produit en Poitou ${ }^{6}$. Il est caractérisé par une bordure périphérique ornée de triangles délimitant, des traverses et une bande longitudinale recouvertes de chevrons consécutifs et ponctuées aux extrémités de cercles. Des sillons rectilignes ou ondulés comblent les vides. L'examen du couvercle, au décor foisonnant, est riche en enseignements pour la connaissance des étapes de fabrication, des techniques, des outils et des gestes. Le couvercle porte de nombreuses traces de pic sur les bandes enserrant les caissons sur le dessus du couvercle et les montants verticaux. Avant de recevoir l'étape de la sculpture, la finition a été exécutée à l'aide d'un ciseau plat, poussé afin de surfacer le parement. Puis le décor a été sculpté à l'aide de deux types d'outils: une broche et un ciseau gravelet pour obtenir une gravure en $\mathrm{V}$ ou en creux, carrée et peu profonde (fig. 2.b). 
Fig. 2. Sarcophage d'Antigny

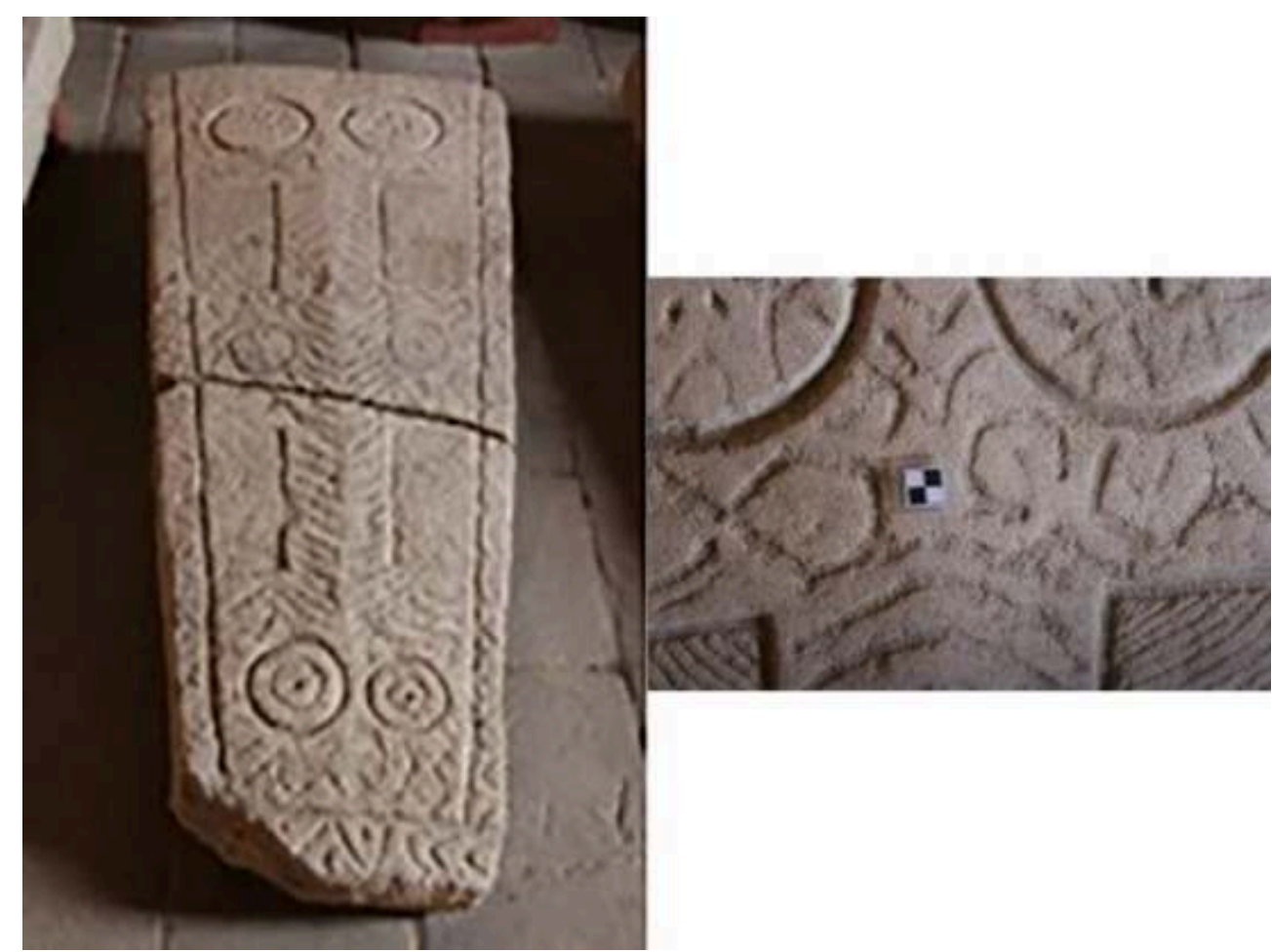

a. Antigny (Vienne), sarcophage 41, couvercle bombé, décor de bandes de stries, formant une croix, et de cercles, VII siècle, calcaire, Poitiers, baptistère Saint-Jean. b. Antigny (Vienne) sarcophage 41, détail du décor offrant trois types de gravure, Poitiers, baptistère Saint-Jean.

(c) T. Grégor (c) T. Grégor

8 Pour réaliser une gravure en $\mathrm{V}$, le graveur tient son outil à $45^{\circ}$ par rapport au plan de frappe et incise la pierre des deux côtés du tracé de la ligne jusqu'à obtenir le V (fig. 3a). Cette gravure en $\mathrm{V}$, qui fait référence aux techniques antiques, est maladroitement exécutée en raison de la complexité du geste ou de l'utilisation d'un outil non adapté. Les traces laissées par le gravelet sont parfaitement visibles et n'ont pas été effacées, les sillons sont perfectibles et la finition est approximative, selon un mode d'exécution que l'on retrouve en général sur les sarcophages locaux.

9 Un second procédé de gravure est caractérisé par une gravure en creux carrée peu profonde, obtenue avec un ciseau gravelet tenu à plat perpendiculairement au plan d'activité afin de tailler peu profondément la surface de la pierre (fig. 3b). Le sculpteur taille en faisant reculer son outil pour obtenir un dessin, qui peut aboutir notamment à des formes arrondies et des cercles, un geste qui présente manifestement des difficultés pour l'artisan de cette période.

Le troisième procédé est atypique. Il s'agit d'une gravure réalisée à la broche, un outil très utilisé dans la taille de pierre et la sculpture au cours de l'Antiquité tardive et du haut Moyen Âge pour les étapes de dégrossi, rarement pour les finitions. Le sculpteur grave de multiples points en heurtant une broche à l'aide d'un percuteur, la succession des points formant des sillons concentriques. Cette méthode est parfois utilisée pour graver les lettres des inscriptions (fig. 3.c). 
Fig. 3. Démonstration, exécution d'une gravure
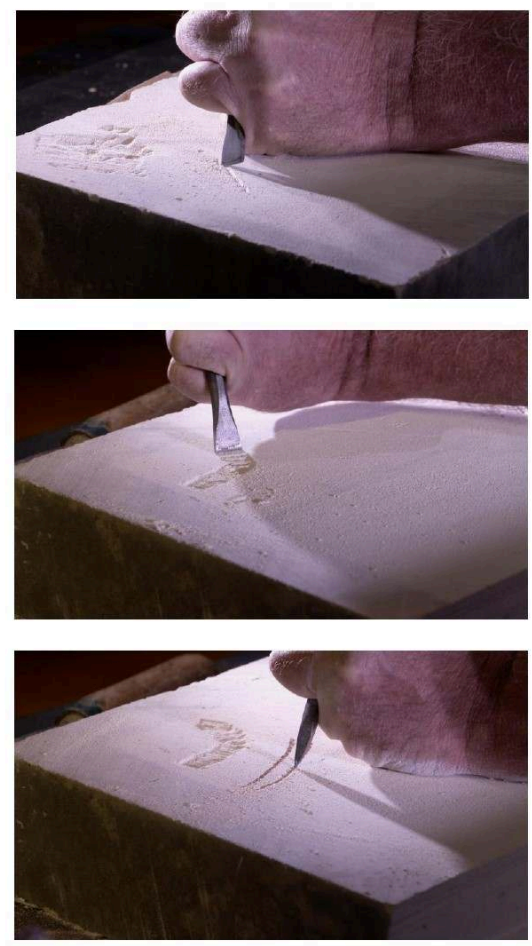

a. Exécution d'une gravure en $\vee$ en démonstration. b. Exécution d'une gravure en creux carrée en démonstration. c. Exécution d'une gravure à l'aide d'une broche en démonstration.

(c) musée du Louvre

\section{La question des surfaces tridimensionnelles}

11 Les sculpteurs travaillant sur des surfaces planes ou sur des surfaces en trois dimensions emploient les mêmes types d'outils, seule la méthode d'approche de la matière et donc le niveau technique diffère.

Les chapiteaux sont représentatifs de la sculpture en trois dimensions. Conservés en nombre relativement important, ils appartiennent à plusieurs types qui s'inspirent tous, au départ, de modèles antiques et offrent des formes décoratives nouvelles. Le chapiteau corinthien est l'élément le plus représentatif de l'architecture romaine. Diffusé en Gaule, il a séduit aussi bien les commanditaires que les architectes par sa richesse décorative: l'élément végétal - feuilles d'acanthe des deux couronnes inférieures, volutes et hélices - se prêtait à l'imagination.

13 Le chapiteau provenant de l'église parisienne Sainte-Croix-et-Saint-Vincent (aujourd'hui Saint-Germain-des-Prés) se rattache au groupe composite, qui a connu très tôt une grande faveur. Il s'agit d'un compromis entre les ordres ionique et corinthien, nouveau schéma qui a été particulièrement prisé au cours de l'Antiquité tardive en raison des possibilités multiples offertes par l'association de certains caractères des deux ordres. La corbeille offre un schéma de composition fondé sur quatre feuilles d'angle à l'origine recourbées sous les volutes, dont les folioles médianes digitées sont tangentes et traitées en taille biseautée. La tige des feuilles est soulignée 
par des bandes ornées de motifs de vannerie à stries obliques alternées. Une croix à l'aplomb du dé médian de l'abaque s'insère entre les crosses sur le calathos (fig. 4.a).

Pour l'exécution de ce chapiteau, comme pour celle du couvercle de sarcophage d'Antigny, le sculpteur a utilisé une broche (fig. 4.b), non pas pour la finition mais pour enlever de la matière et réaliser le travail d'approche de la sculpture. Les traces de broche sont encore discernables sur le revers (fig. 4.c), car il n'a pas pris la peine de surfacer les parties cachées par les folioles. Économie d'effort et économie d'outillage guident son travail, avec une idée précise de la position finale de l'œuvre dans l'espace architectural et de l'angle de vue.

Fig. 4. Chapiteau du musée du Louvre

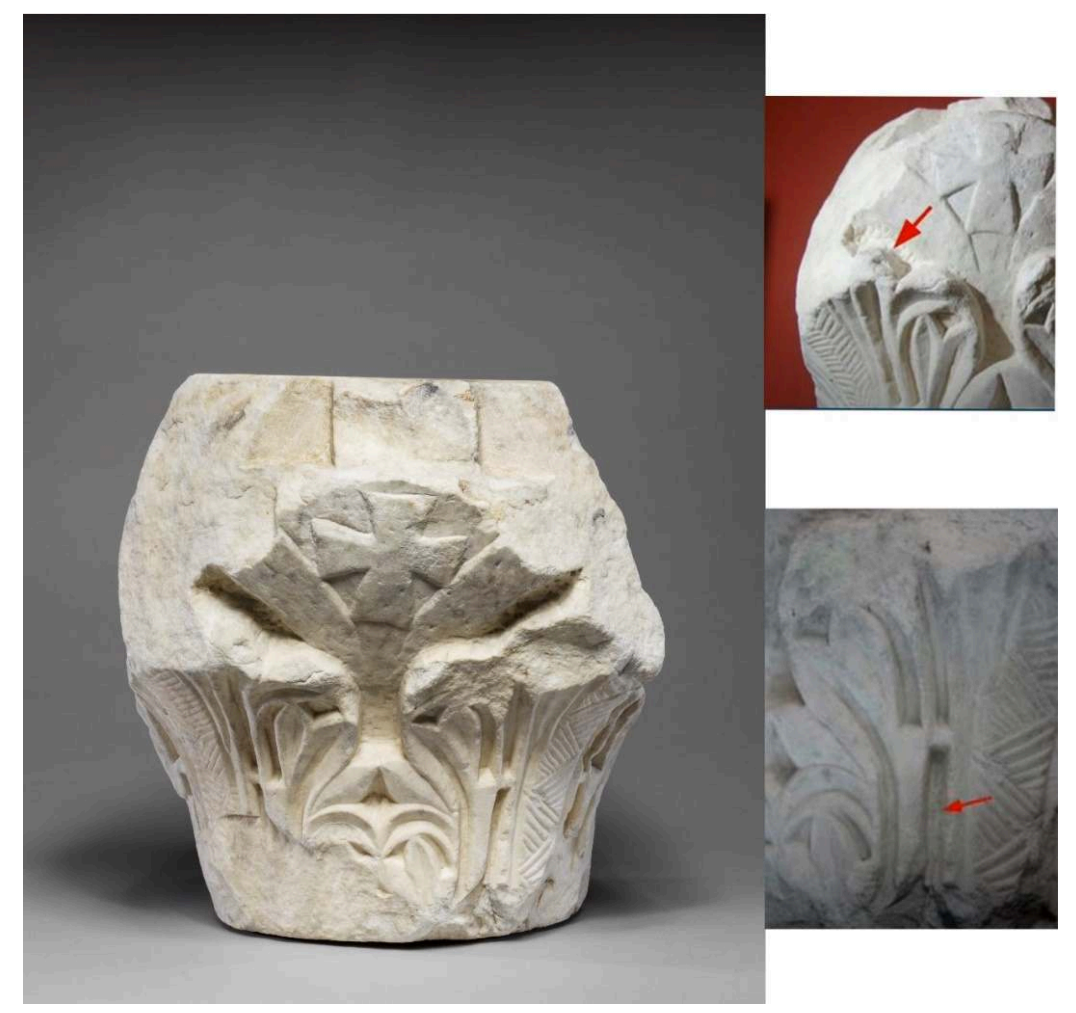

a. Chapiteau composite orné de feuilles d'acanthe, de motifs de vannerie et d'une croix pattée, $V^{e}$ siècle église Sainte-Croix-et-Saint-Vincent fondée par le roi Childebert (v. 559), Ve siècle, marbre, H. : $0,28 \mathrm{~m} ;$ । . : 0,305 m ; Pr. : 0,29 m, Paris, musée du Louvre, inv. MNC 410. b. Chapiteau composite orné de feuilles d'acanthe MNC 410, détail de la sculpture avec un ciseau gravelet sur le chapiteau. C. Chapiteau MNC 410 : détail des traces de broche.

(c) 2017 Musée du Louvre / Philippe Fuzeau @ musée du Louvre @ musée du Louvre

Une fois le bloc dégrossi, l'exécutant a utilisé un ciseau gravelet pour la sculpture ellemême, et a procédé de deux façons pour la finition : la première, la plus classique, en faisant des incisions en $\mathrm{V}$, l'outil posé à $45^{\circ}$ par rapport au plan de frappe comme sur le sarcophage d'Antigny; la seconde, plus laborieuse, consistant à tenir l'outil à plat pour obtenir des incisions carrées profondes et larges. Cette opération s'avère techniquement plus ou moins difficile en fonction des matériaux, marbre ou calcaire, car l'outil, au fur et à mesure qu'il est percuté, pénètre dans la matière et a tendance à se bloquer (fig. 5). 
Fig. 5. Démonstration, exécution d'une sculpture
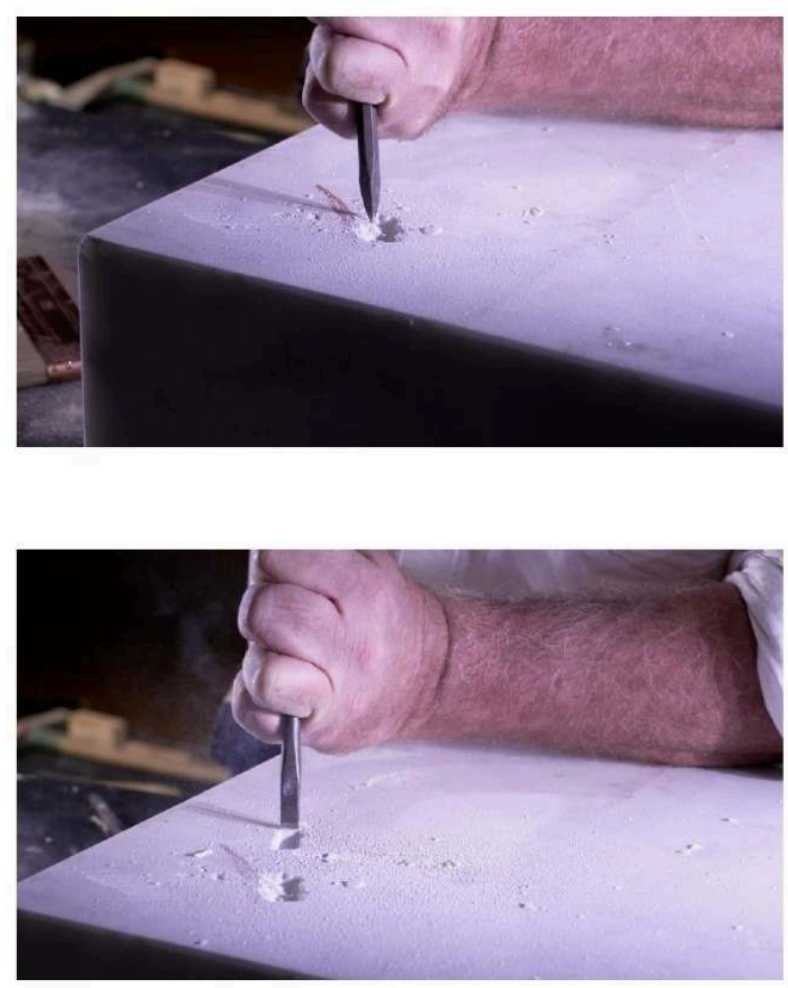

a. Exécution d'une sculpture avec une broche dans du marbre, démonstration. b. Exécution d'une sculpture avec un ciseau gravelet, démonstration.

(c) musée du Louvre

Les deux chapiteaux suivants, en marbre des Pyrénées, au musée du Louvre (RF 3332 et RF 3333), permettent d'aborder d'autres questions. Ils illustrent deux variantes du groupe dérivé du corinthien. Le premier, à deux rangs d'acanthes, est caractérisé par la modification du registre supérieur: aux composants classiques - gaine, collerette, et calice - se substituent des feuilles stylisées traitées en biseau et débordant sur l'abaque, créant une fusion entre ces deux parties (fig. 6a). Sur le second, à deux rangs d'acanthes, le sculpteur a supprimé le registre des calices (fig. 6b). Le rang inférieur est composé de huit feuilles d'acanthe d'où s'échappent les feuilles d'angle encadrant une palmette médiane grossièrement taillée sur le calathos. Les nervures plates des feuilles sont creusées en gouttière et les folioles traitées en " pince de crabe ». Sur l'abaque, au profil profondément découpé et aux angles abattus, les volutes partent à l'oblique de la base du dé axial saillant. Par le matériau, marbre des Pyrénées, et le traitement, ils s'apparentent à la production des ateliers du Sud-Ouest de la France. 
Fig. 6. Chapiteau conservé au Louvre

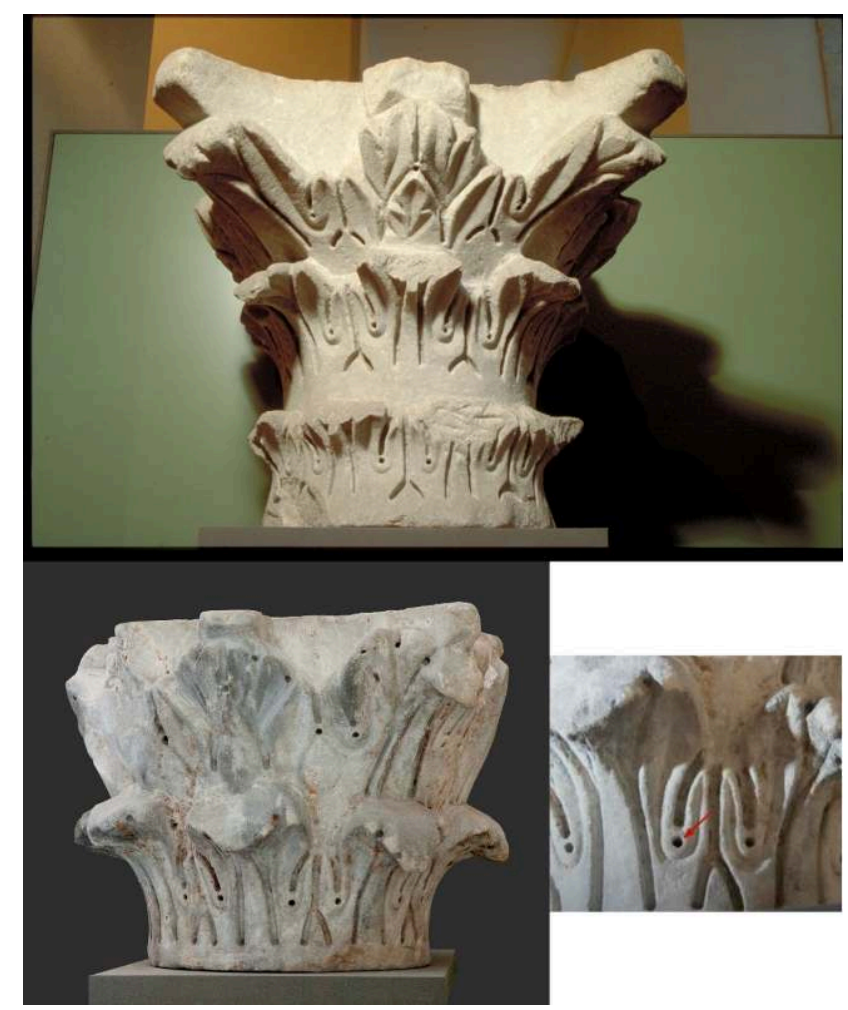

a. Chapiteau dérivé du corinthien à double couronne de feuilles d'acanthe, provenance inconnue, Ve siècle, marbre, H. : 0,285 m ; I. : 0,41 m ; Pr. : 0,415 m, Paris, musée du Louvre, RF 3333. b. Chapiteau dérivé du corinthien à double couronne de feuilles d'acanthe, provenance inconnue, $V$ e siècle, marbre, H. : 0,36 m ; I. : 0,42 m ; Pr. : 0,415 m, Paris, musée du Louvre, RF 3332. c. Chapiteau RF3333 : détail de la sculpture avec un foret.

(C) 1978 Réunion des musées nationaux - Grand Palais (c) 1978 Réunion des musées nationaux - Grand Palais (c) musée du Louvre

Les techniques d'exécution sont semblables à celles du chapiteau MNC 410. Cependant, aux traces de broche et au travail au ciseau gravelet s'ajoute l'utilisation du foret avec mèche afin de façonner les moulures, d'accentuer les reliefs. Le sculpteur réalise une perforation à l'aide du foret (fig. 6c), qui lui permet soit de positionner des points de repère précis avant de sculpter et ensuite de faire uniquement la perforation terminale (fig. 7), soit de réaliser un canal. Les perforations peuvent également être exécutées avec un tamponnoir (fig. 7.b), par percussion et rotation entre les coups pour creuser la pierre. 
Fig. 7. Exécution d'une sculpture, démonstration
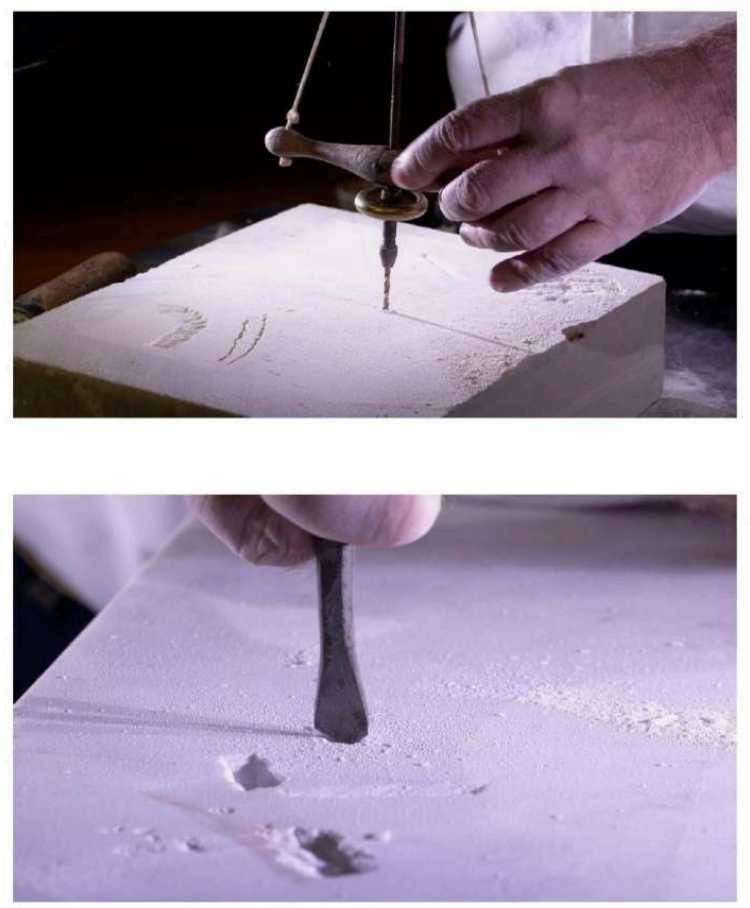

a. Exécution d'une sculpture avec un foret dans un bloc de calcaire, démonstration. b. Exécution d'une sculpture avec un tamponnoir dans un bloc de marbre, démonstration.

(c) musée du Louvre

Comme sur les œuvres précédentes, des traces de broche, de ciseau gravelet et de trous de petites mèches sont visibles sur l'un des deux chapiteaux en marbre des Pyrénées tardo-antiques (RF 3332) (fig. 8). Or ce chapiteau montre une spécificité : l'emploi d'un ciseau gravelet à bout-rond (fig. 8 et fig. 9). Ce type d'outil est rarement utilisé, même s'il est présent parfois sur des éléments de sculpture antique. Le ciseau gravelet, généralement doté d'une partie active droite, possède dans ce cas une partie active arrondie, plus efficace pour excaver les fonds de moulure. Ceux-ci ont d'abord été creusés à l'aide d'un ciseau gravelet droit, puis ont été achevés au ciseau gravelet boutrond afin d'approfondir le canal. 
Fig. 8. Chapiteau, détail

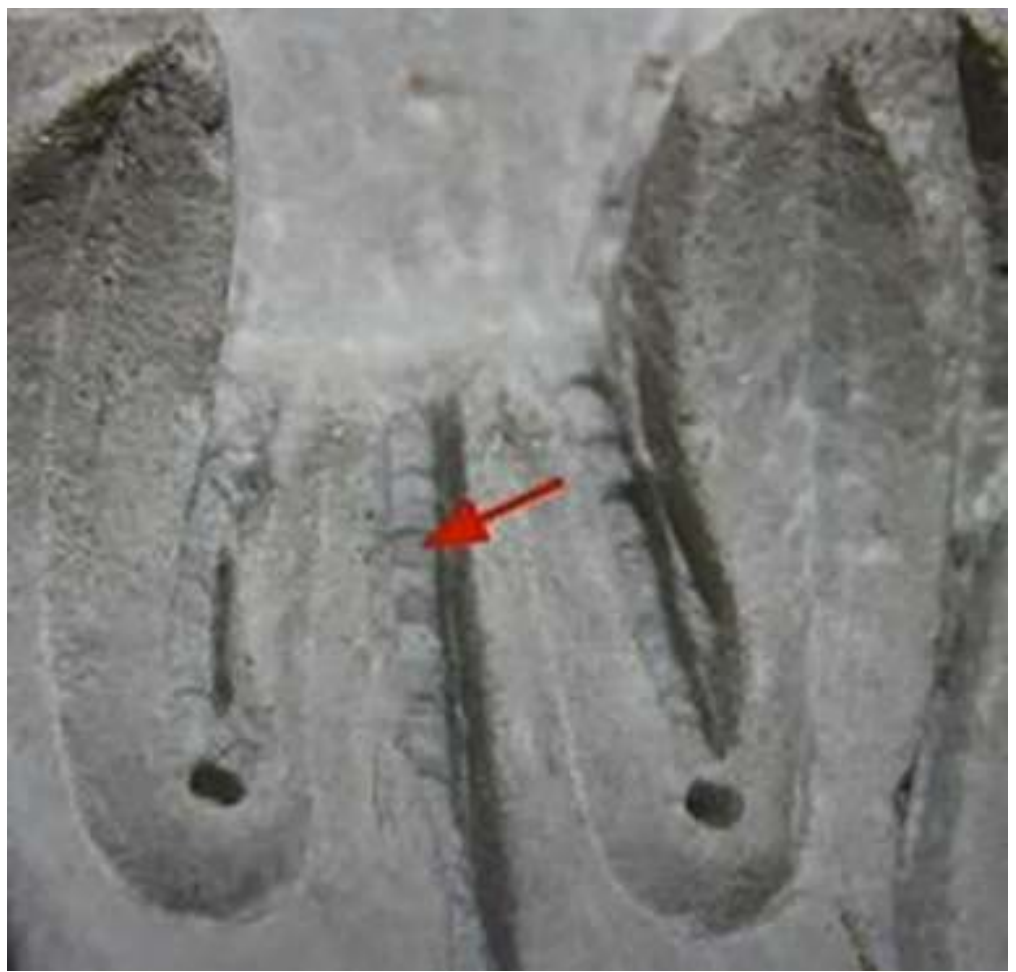

Chapiteau RF 3332, détail de la trace la sculpture réalisée avec un bout-rond. (c) musée du Louvre 
Fig. 9. Exécution d'une sculpture, démonstration
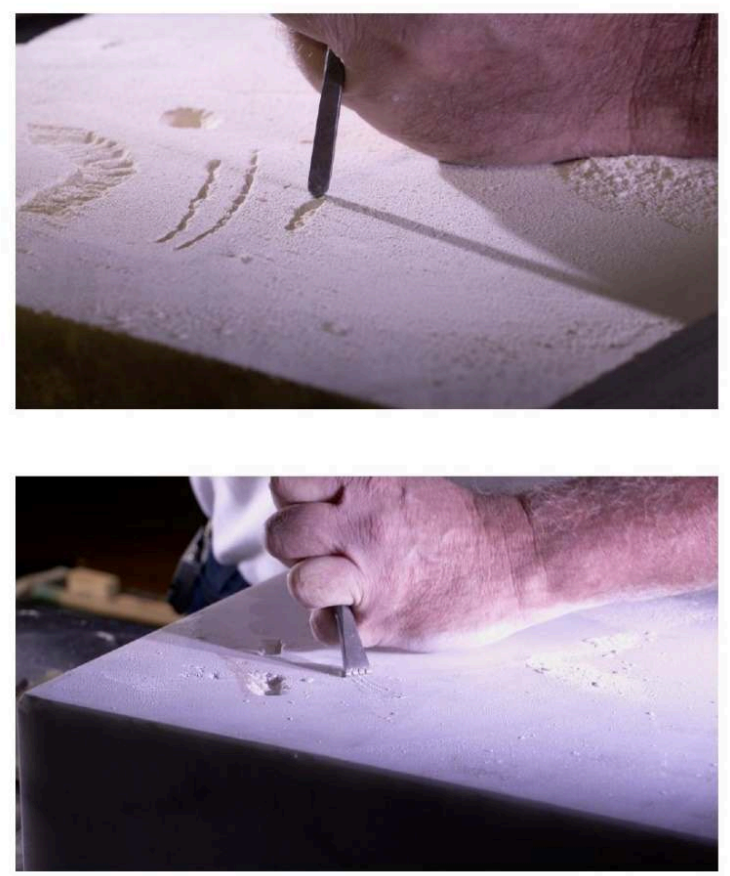

a. Exécution d'une sculpture avec un ciseau bout-rond, démonstration. b. Exécution d'une finition avec une gradine, démonstration.

(c) musée du Louvre

À la ripe, outil à percussion posée, sans percuteur, qui est utilisé le manche tenu dans une main, l'autre main appuyant fortement sur la partie active de la ripe, l'action d'usure finissant les surfaces sont visibles. Ainsi, le bloc a été taillé à l'aide d'une broche et d'un ciseau puis surfacé à la ripe. Un autre type de finition est envisageable, le surfaçage du parement à l'aide d'un ciseau, gravelet ou gradine (fig. 9.b).

Le dernier chapiteau analysé au musée du Louvre (RF 457) offre la singularité d'avoir une face sculptée au $\mathrm{V}^{\mathrm{e}}$ siècle, l'autre resculptée au XII siècle et d'être en marbre de Carrare. La face à décor d'acanthes épineuses tardo-antique (fig. 10.a) présente la même technique de taille que les œuvres précédentes. Le bloc a été épannelé, puis des trous ont été percés avec un foret. Le sculpteur a souligné avec un ciseau gravelet fin de $3 \mathrm{~mm}$, les filets, puis avec un ciseau gravelet de $8 \mathrm{~mm}$ pour les surfaces planes et concaves. Méplats et traces d'outils restent visibles sur les parties sculptées, la finition étant imparfaite. La face ornée du thème de Daniel dans la fosse aux lions a été traitée en haut relief (fig. 10.b). Les marques de dégagement des formes apparaissent derrière le lion de gauche. Le reste d'une petite ciselure ainsi que les traces d'un ciseau plutôt large utilisé pour préparer les fonds sont perceptibles (fig. 10.c). Le sculpteur s'est servi d'un petit ciseau gravelet pour réaliser la ciselure puis a changé d'outil, prenant un ciseau plus large pour enlever la matière (fig. 10.d), un outil peu efficace et non adapté au marbre. 

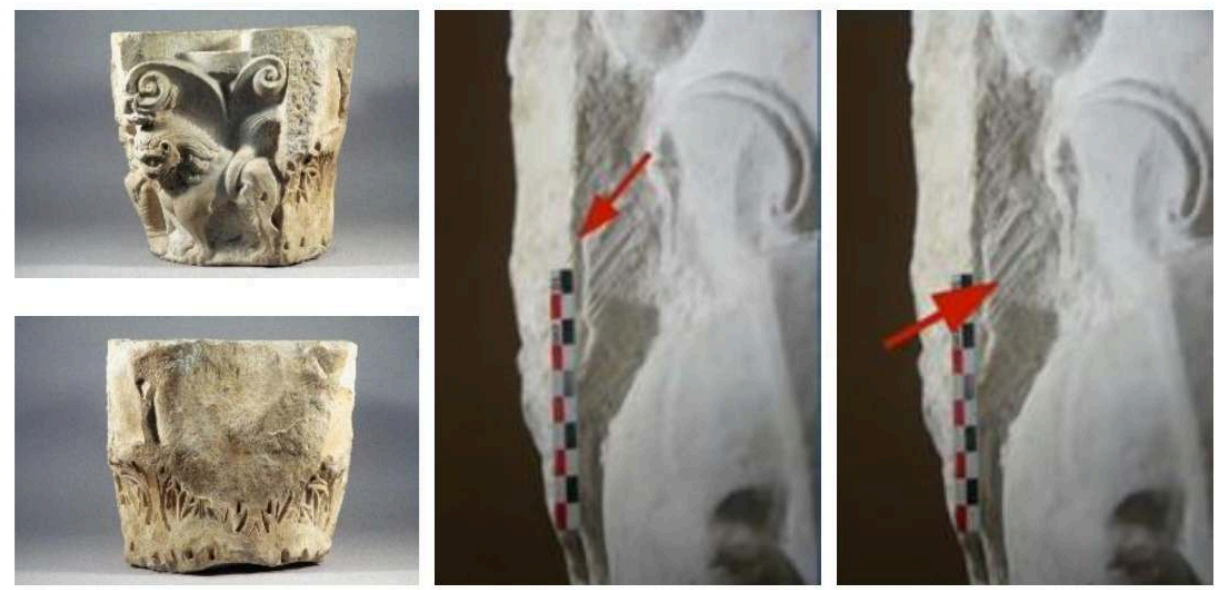

a. Chapiteau à décor végétal tardo-antique, dégagé dans l'emprise de l'église des Saints-Apôtres (église Sainte-Geneviève), Paris, resculpté d'une figuration de Daniel dans la fosse aux lions au XIIe siècle, marbre de Carrare, H. : 0,495 m ; I. : 0,53 m ; Pr. : 0, 51 m, Paris, musée du Louvre, RF 457. b. Chapiteau RF 457, Daniel dans la fosse aux lions (XII e siècle). c. Chapiteau RF 457, Daniel dans la fosse aux lions : détail de la ciselure traitée au ciseau gravelet. d. Chapiteau RF 457, Daniel dans la fosse aux lions : détail du dégrossi.

(c) 2004 musée du Louvre / Pierre Philibert (c) musée du Louvre @ 2004 Musée du Louvre / Pierre Philibert @ 2004 musée du Louvre / Pierre Philibert

Des traces de broche sont également présentes sur les parties qui ne devaient pas être visibles une fois le chapiteau mis en place, toujours pour respecter la règle d'économie de temps et de moyens, puis le reste de la sculpture a été certainement réalisé avec un ciseau gravelet fin. Cette partie du bloc semble taillée par un exécutant connaissant les techniques de sculpture mais peu, voire pas, ce matériau spécifique, le marbre; il s'agit donc d'un excellent sculpteur du nord de la France, travaillant le calcaire et découvrant le marbre. Ainsi, on observe deux approches, sur un même matériau - le marbre -, à des époques distinctes par deux sculpteurs qui ont réalisé des œuvres totalement différentes : la face végétale est traitée en faible relief, les volumes sont juste marqués par des incisions et des plans légèrement inclinés, alors que sur la face du XII ${ }^{e}$ siècle, il n'a pas hésité à entrer dans la matière, à travailler en haut-relief pour obtenir une sculpture de grande qualité plastique et esthétique.

\section{Ateliers et production}

L'observation de deux autres plaques sculptées dans divers matériaux, créées par des ateliers différents et répondant à des commandes spécifiques, met en évidence la disparité de la production haut médiévale.

La première, en calcaire, est un bloc trapézoïdal monolithe surmonté d'un élément vertical dont seule est visible la base tronquée identifiée à un socle d'une crucifixion ${ }^{7}$ (fig. 11). Il s'agit de la pierre dite « des larrons » conservée au musée Sainte-Croix de Poitiers et découverte par le père de La Croix, avec d'autres blocs, dans l'hypogée des Dunes de la même ville. Datée du VIII ${ }^{e}$ siècle, elle présente la figuration des deux 
personnages masculins placés de part et d'autre d'une colonne, devant les montants horizontaux de leurs croix.

Fig. 11. Base ou socle d'une crucifixion

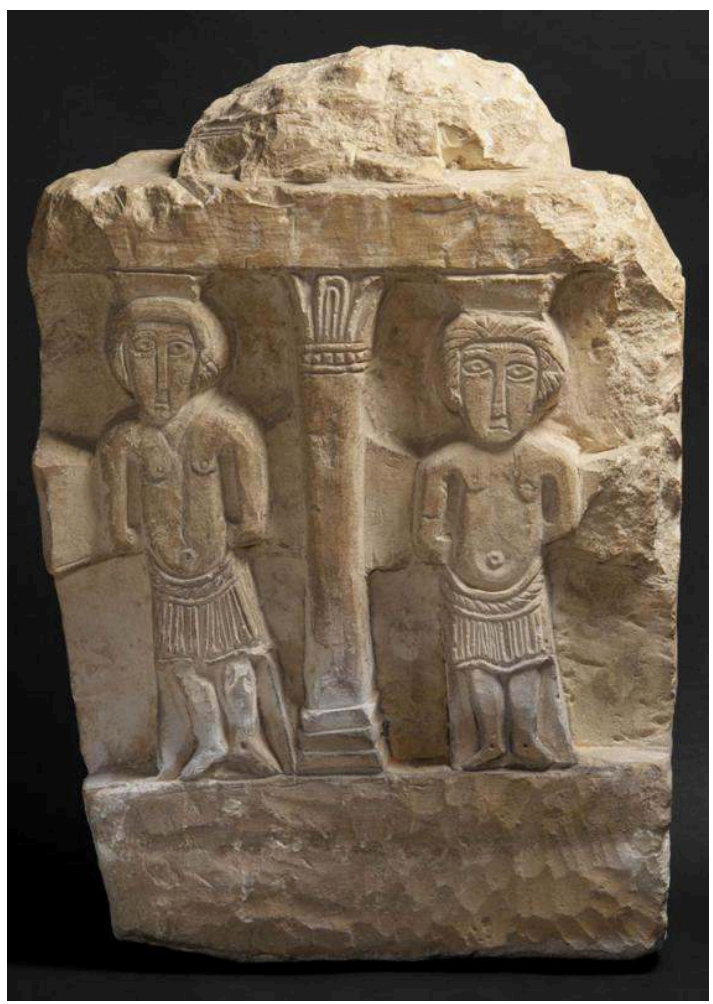

Base ou socle d'une crucifixion, Poitiers, hypogée des Dunes, début VIII siècle, calcaire, H. :0,64 m ; I. : 54/46 m ; Pr. :0,16/0,22 m, Poitiers, musée Sainte-Croix.

(c) Musée de Poitiers, Ch. Vignaud

Un des premiers détails visibles sur le bloc est la taille ou retaille à l'aide d'un outil à percussion lancée, certainement un têtu-taillant, type d'outil utilisé pour enlever de la matière lors des premières étapes de la taille de pierre ou de la sculpture (fig. 12). 
Fig. 12. Base ou socle d'une crucifixion

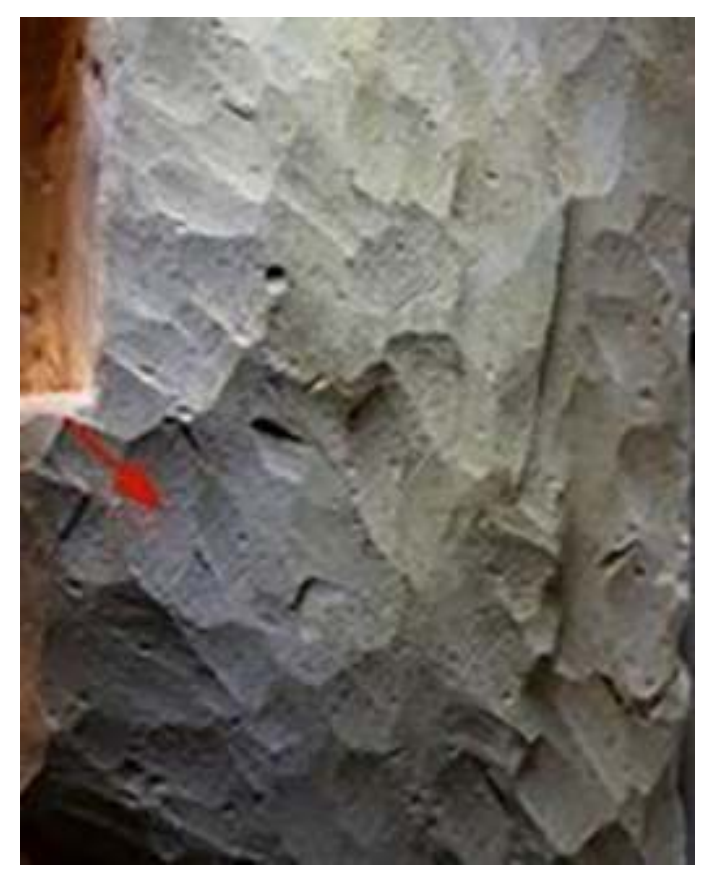

Base ou socle d'une crucifixion, Poitiers, hypogée des Dunes, détail des ocelles.

(c) musée du Louvre

Sur le relief, le têtu-taillant utilisé a la particularité d'être ébréché et a laissé des traces spécifiques sur les plans de joints verticaux, en bas du socle et en partie supérieure, marques visibles également sur un grand nombre de blocs conservés dans l'hypogée des Dunes (fig. 13).

Fig. 13. Taille de pierre, démonstration

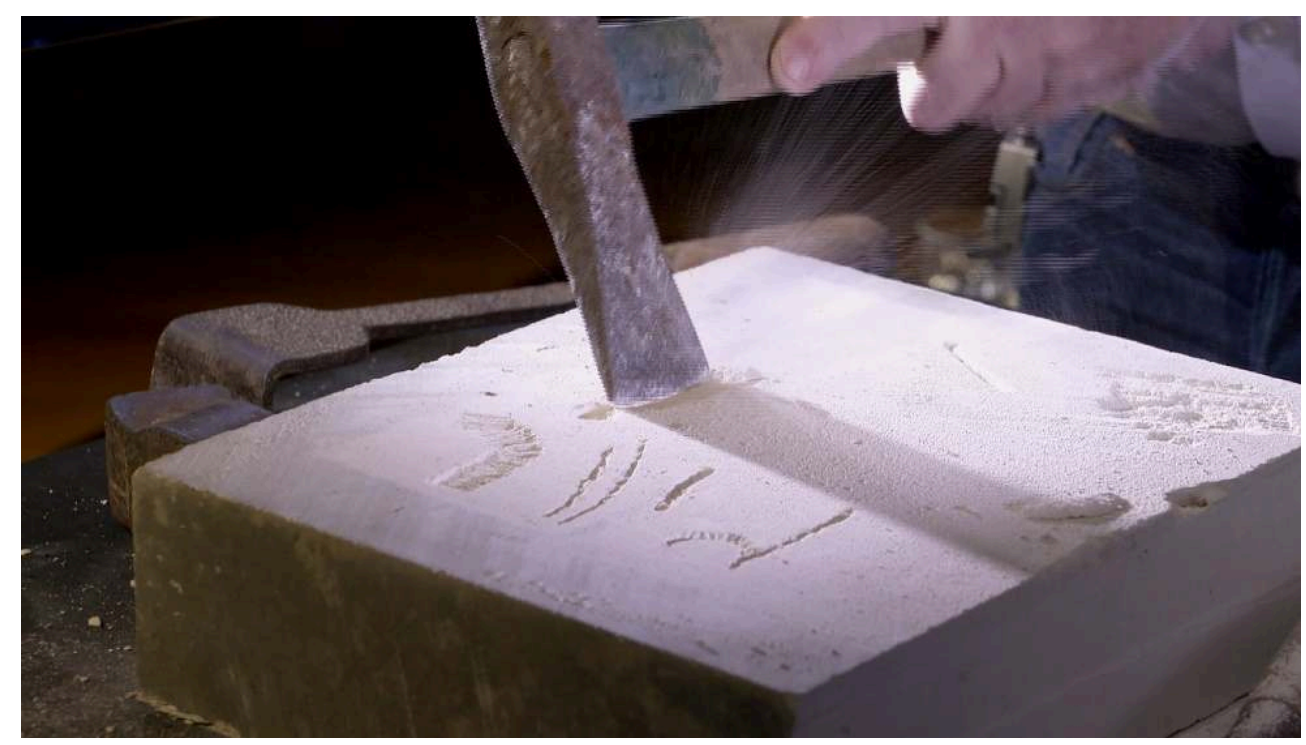

Taille de pierre réalisée avec un têtu-taillant, démonstration.

(c) musée du Louvre

26 La sculpture a été réalisée en «moyen-relief » ou " demi-relief »; les fonds présentent quelques traces éparses de broche, le surfaçage semble avoir été réalisé à l'aide d'un 
ciseau tenu à plat. Le sculpteur a rencontré manifestement des difficultés pour la finition des amortis entre la croix et les personnages. Les parties sculptées elles-mêmes offrent de nombreux méplats, résultant d'un travail de la pierre sans percussion, par incision et grattage à l'aide d'un ou plusieurs outils du type ciseau.

Un détail montre l'ingéniosité de l'exécutant. Les nombrils ont vraisemblablement été travaillés à l'aide de ce que l'on peut appeler un « outil » pour tracer des cercles ou des ocelles, une plaquette en bois avec des pointes, car la gravure de cercles concentriques de petit rayon avec un compas relève du défit en raison de la longueur de l'outil et des contorsions de l'exécutant pour créer de nombreux cercles identiques. L'utilisation de plaquette à trois pointes permet de tracer des cercles parfaitement identiques qui peuvent être retravaillés aisément sur un plan vertical (fig. 14).

Fig. 14. Ocelles
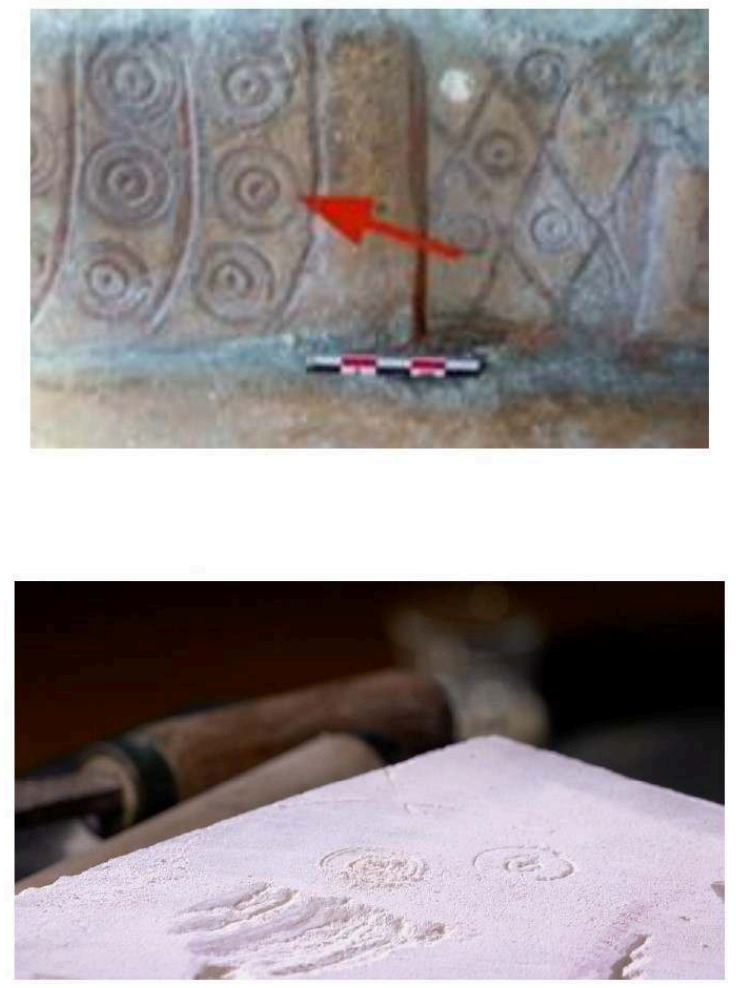

a. Ocelles. b. Technique du tracé des ocelles, démonstration.

(c) musée du Louvre ( musée du Louvre

Le relief en marbre, dit «tombeau du Ladre ", qui provient de l'abbaye de Saint-Denis (fig. 15), laisse percevoir une adresse supérieure du sculpteur. Il atteint ici la perfection non seulement dans l'exécution des motifs ornementaux, d'un grand classicisme, mais également dans la finition du relief par polissage. Un œil averti distingue cependant quelques traces des différentes perforations (fig. 16), ainsi que d'infimes traces d'un seul outil, la ripe. 
Fig. 15. Tombeau du Ladre

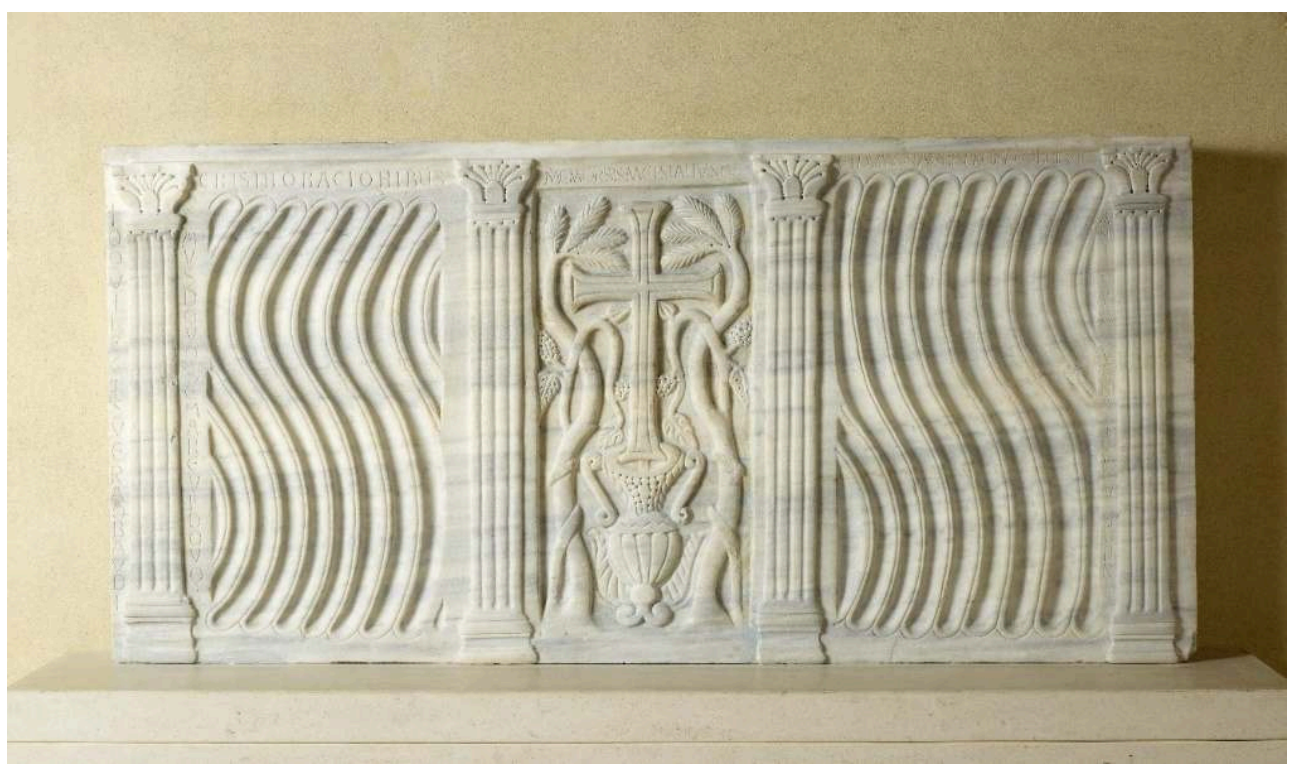

Relief décoré de pilastres, de strigiles et d'un vase surmonté d'une croix dit « tombeau du Ladre », provenance abbaye de Saint-Denis, VIII siècle, marbre, H. : 0,98 m ; L. : 2,02 m ; Pr. : 0,15 m, Paris, musée du Louvre, inv. MNB 3270.

(c) 2018 Musée du Louvre / Anne Chauvet

Fig. 16. Tombeau du Ladre, détail

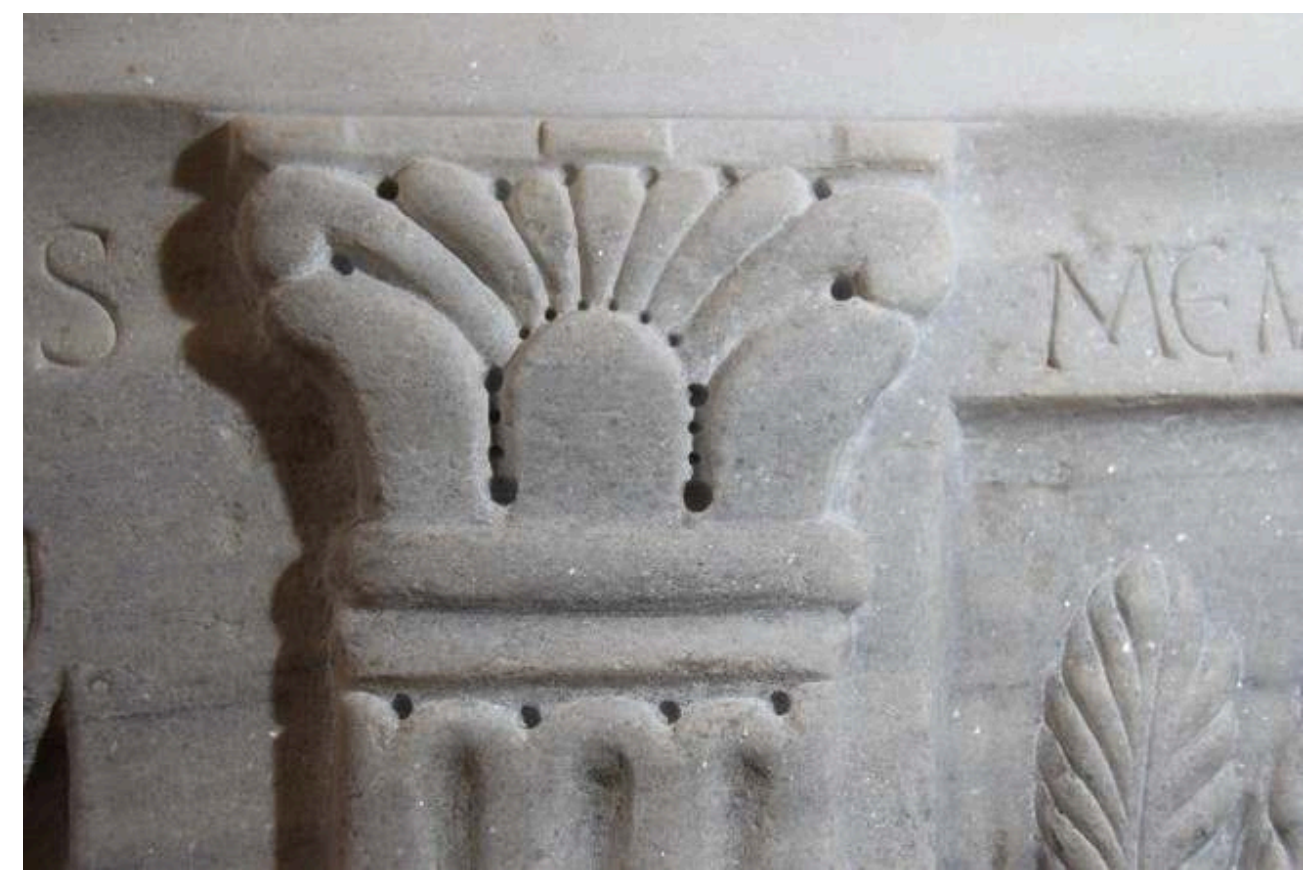

Détail du chapiteau du pilastre dit « tombeau du Ladre ».

(C) T. Grégor

La majorité des œuvres sculptées au cours de l'Antiquité tardive et du haut Moyen Âge se démarque de la production antique. Les traces laissées sur les parements attestent l'usage d'outils basiques et simples - broches et ciseaux - types d'outils usuels dans l'Antiquité, maniés avec moins de dextérité, parfois à mauvais escient, les finitions 
étant peu soignées. Ces observations sur l'altération de la qualité, l'évolution vers la platitude du relief, l'iconographie simplifiée ont suscité nombre d'interprétations: perte de savoir-faire, déstructuration d'une profession ou simplement transformation du goût des commanditaires, moins exigeants? Quoiqu'il en soit ces phénomènes peuvent être liés à une évolution du travail de la pierre, avec une spécialisation moindre des artisans et la disparition des distinctions encore mentionnée dans l'Édit du Maximum édicté par Dioclétien, régulant les salaires au début du $\mathrm{IV}^{\mathrm{e}}$ siècle notamment pour les carriers, tailleurs de pierre, bronziers, sculpteurs, lapicides. Malgré ces remarques un peu dépréciatives, il faut souligner que les exécutants réalisent des œuvres pleines de vie et qui n'ont rien à envier, par leurs qualités propres, à leurs prédécesseurs de l'Antiquité classique.

\section{NOTES}

1. Jean-Claude Bessac. L'outillage traditionnel du tailleur de pierre de l'Antiquité à nos jours, Paris, C.N.R.S., 1987. Voir notamment p. 319.

2. Étienne Cunrath, Pierre de taille, Artisanat - Outillage et applications : Forge - Extraction Taille, Tours, Archives des artisans de la Pierre de Taille, 2007. Voir notamment p. 272.

3. Ouvrage collectif, Encyclopédie des métiers: La maçonnerie et la taille de pierre, Paris, Librairie du compagnonnage, 2019.

4. Les clichés : 1a, 1b, 1c, 2a et $2 b, 3 a, 4 c, 5 b, 6 b$ et $6 c, 7 a, 8$, 9a et 9b, 10c, 10d, 12, 13, 14a, sont issus de la captation par les services du Louvre de l'intervention du 25 septembre 2020 lors du colloque "Classique et Barbare », le lien de visionnage étant visible sur https://www.youtube.com/watch? $\mathrm{v}=\mathrm{x} 7$ CkGEH3i8k [dernière consultation le 09/11/2021].

5. Margherita Coppola et Anne Flammin, «Les sarcophages au musée lapidaire du baptistère Saint-Jean de Poitiers : classement typologique et étude iconographique », Bulletin de la société des antiquaires de l'Ouest et des musées de Poitiers, Tome VIII, $3^{\mathrm{e}}-4^{\mathrm{e}}$ trimestres, (1994) $\mathrm{n}^{\mathrm{os}} 3-4$, 1997.

6. Guillaume Rougé, Les sarcophages entre Loire et Pyrénées: observations et études par des critères, techniques et morphologiques, thèse, Université Michel de Montaigne - Bordeaux III, 2014. HAL https://tel.archives-ouvertes.fr/tel-01531836. Voir p. 137 (Groupe D-6, Décor de sillons).

7. Camille de La Croix, Monographie de l'hypogée-martyrium de Poitiers, Paris, Librairie de Firmin Didot, 1883, p. 24.

\section{RÉSUMÉS}

L'Antiquité tardive et le haut Moyen Âge présentent des spécificités techniques quant à la sculpture sur pierre. En mettant en regard diverses œuvres du musée du Louvre et du musée 
Sainte-Croix de Poitiers avec les conditions d'utilisation des outils, parfois particuliers et originaux, la communication détaille ces techniques spécifiques et l'approche du matériau pierre, toujours ingénieux, des graveurs, sculpteurs, tailleurs de pierre ou tout simplement des exécutants au cours de cette période charnière.

Stone-carving techniques in Late Antiquity and the Early Middle Ages were very specific. By comparing various works from the Louvre and the Musée Sainte-Croix in Poitiers with the conditions of use of the tools, sometimes particular and original, the paper will describe in detail these specific techniques and the approach to the stone material, always ingenious, of the engravers, sculptors, stone cutters or simply the executors during this pivotal period.

\section{INDEX}

Keywords : technique, sculptor, Late Antiquity, Early Middle Ages, tools, marble stones, limestone

Mots-clés : technique, sculpteur, antiquité tardive, haut Moyen Âge, outils, pierres marbrières, calcaire

\section{AUTEURS}

\section{THIERRY GREGOR}

Thierry Grégor est doctorant au Centre d'Etudes Supérieures de Civilisation Médiévale de Poitiers sous la direction de Cécile Treffort avec pour sujet « Les inscriptions lapidaires antiques et médiévales : vers une approche technique de leur réalisation ». Tailleur de pierre, enseignant, il a participé et participe à des missions de fouilles à l'étranger sur des sujets techniques concernant la pierre, notamment dans le bassin méditerranéen et au Moyen-Orient.

Thierry Grégor is preparing his doctoral thesis, "Les inscriptions lapidaires antiques et médiévales : vers une approche technique de leur réalisation", at the Centre d'Études Supérieures de Civilisation Médiévale de Poitiers, under the supervision of Cécile Treffort. A stone cutter and teacher, he takes part in excavations abroad involving stone, particularly in the Mediterranean basin and the Middle East.

\section{ANNE-BÉNÉDICTE MÉREL-BRANDENBURG}

Membre de l'équipe de recherche de l'École du Louvre, Anne-Bénédicte Mérel-Brandenburg après un cursus académique à Paris4-Sorbonne, à l'Ecole du Louvre et à l'EPHE $4{ }^{\mathrm{e}}$ section s'est spécialisée en archéologie de l'Antiquité tardive et du haut Moyen Âge, plus particulièrement en France méridionale, de « l'archéologie funéraire et la culture matérielle en Languedoc méditerranéen » (DEA), à « La sculpture de l'Antiquité tardive et du haut Moyen Âge en Languedoc méditerranéen et Roussillon » (thèse Paris IV-Sorbonne, 2007). Elle a dirigé des chantiers de fouilles archéologiques, un projet collectif de recherche (PCR) sur le baptistère Saint-Jean au Puy-en-Velay, monté un programme de recherche sur la provenance et l'identification des marbres blancs en France et publié les résultats de ses recherches. En 2017, elle a initié le projet d'un Corpus numérique de la sculpture de l'Antiquité tardive et du haut Moyen Âge (SATHMA), un partenariat entre l'École du Louvre, le département des Sculptures du Louvre et le CNRS (UMR 5138 Archéométrie et Archéologie ; ARTEHIS UMR 6298), à l'origine des Journées d'étude qui se sont déroulées à l'Ecole du Louvre et à l'auditorium du Louvre en septembre 2020. À l'École du Louvre, elle a enseigné l'Histoire de l'architecture occidentale, 
Antiquité tardive et haut Moyen âge (CS 1989-2005), l'Archéologie de la Gaule romaine (2014-2017-CO-CS), l'Histoire de la Sculpture (2020-2021) et encadré les travaux pratiques en Archéologie de la Gaule et du premier Moyen âge (2008-2016).

Anne-Bénédicte Mérel-Brandenburg, a member of the École du Louvre research team, studied at the Université de Paris 4-Sorbonne, the École du Louvre and the École Pratique des Hautes Études (Sciences Historiques et Philologiques), specialising in the archaeology of Late Antiquity and the Early Middle Ages, especially that of southern France: the title of her postgraduate dissertation is "L'archéologie funéraire et la culture matérielle en Languedoc méditerranéen" and that of her $\mathrm{PhD}$ thesis is "La sculpture de l'Antiquité tardive et du haut Moyen Âge en Languedoc méditerranéen et Roussillon" (Paris 4-Sorbonne, 2007). She has overseen archaeological excavations and a joint research project on the Baptistère Saint-Jean in Puy-en-Velay, set up a research programme on the provenance and identification of types of white marble in France and published the results of her research. In 2017 she initiated the Corpus Numérique de la Sculpture de l'Antiquité Tardive et du Haut Moyen Âge (SATHMA) project, a partnership between the École du Louvre, the Sculpture Department at the Louvre, and the CNRS (UMR 5138 Archéométrie et Archéologie; ARTEHIS UMR 6298), which led to the study days held at the École $\mathrm{du}$ Louvre and the Louvre auditorium in September 2020. At the École du Louvre, she has taught the history of Western architecture, Late Antiquity and the Early Middle Ages (1989-2005), the archaeology of Roman Gaul (2014-17) and the history of sculpture (2020-21), and has supervised practicals in the archaeology of Gaul and the Early Middle Ages (2008-16). 